\title{
Entropic Functionals in Quantum Statistical Mechanics
}

\author{
Vojkan Jakšić ${ }^{1}$ and Claude-Alain Pillet ${ }^{2}$ \\ ${ }^{1}$ Department of Mathematics and Statistics \\ McGill University \\ 805 Sherbrooke Street West \\ Montreal, QC, H3A 2K6, Canada \\ ${ }^{2}$ Aix-Marseille Université, CNRS UMR 7332, CPT, 13288 Marseille, France \\ Université du Sud Toulon-Var, CNRS UMR 7332, CPT, 83957 La Garde, France
}

To appear in:

Proceedings of the XVIIth International Congress of Mathematical Physics, Aalborg, Denmark, 2012

\begin{abstract}
We describe quantum entropic functionals and outline a research program dealing with entropic fluctuations in non-equilibrium quantum statistical mechanics.
\end{abstract}

\section{Introduction}

Starting with papers $[7,8,18,19]$, the mathematical theory of non-equilibrium quantum statistical mechanics has developed rapidly in the last decade. The initial developments concerned the theory of non-equilibrium steady states, the entropy production observable, and linear response theory (GreenKubo formulas, Onsager reciprocity relations) for open systems driven by thermodynamical forces (say temperature differentials). This line of development was a natural direct quantization of the classical theory. In contrast, extensions of the classical fluctuation relations of Evans-Searles [4] and GallavottiCohen [6] to the quantum domain have led to some surprises and novel classes of entropic functionals with somewhat striking mathematical structure and physical interpretation.

A pedagogical introduction to our research program dealing with fluctuation theorems/relations in nonequilibrium quantum statistical mechanics can be found in [12]. This note can be viewed as a brief introduction to [12]. We sketch in telegraphic and simple terms the finite time/finite volume theory in the classical case (for comparison purposes) and in the quantum case, and comment on the resulting research program. The interested reader may consult $[13,12]$ for additional information. 


\section{Classical picture}

Consider a pair $(\mathcal{E}, \phi)$, where $\mathcal{E}=\left\{\zeta_{j}\right\}_{0 \leq j \leq N}$ is a finite phase space and $\phi: \mathcal{E} \rightarrow \mathcal{E}$ is a discrete time dynamics. For our purposes without loss of generality we may assume that $\phi\left(\zeta_{j}\right)=\zeta_{j+1}\left(\zeta_{N+1}=\zeta_{0}\right)$. Observables are functions $f: \mathcal{E} \rightarrow \mathbb{R}$ and states are non-vanishing probability measures $\rho$ on $\mathcal{E}$. We write $\rho(f)=\sum_{\zeta} f(\zeta) \rho(\zeta)$. Observables evolve in time as $f_{t}=f \circ \phi^{t}, t \in \mathbb{Z}$, and states as $\rho_{t}(f)=\rho\left(f_{t}\right)$.

The relative entropy of two states

$$
S(\rho, \nu)=\sum_{\zeta} \rho(\zeta) \log (\nu(\zeta) / \rho(\zeta))
$$

satisfies $S(\rho, \nu) \leq 0$ and $S(\rho, \nu)=0$ iff $\rho=\nu$. The Rényi relative entropy of order $\alpha \in \mathbb{R}$ is defined by

$$
S_{\alpha}(\rho, \nu)=\log \sum_{\zeta} \rho(\zeta)^{1-\alpha} \nu(\zeta)^{\alpha}
$$

Our starting point is a dynamical system $\left(\mathcal{E}, \phi, \omega_{0}\right)$ where $\omega_{0}$ is a given reference state. We assume that $\omega_{0}$ is not constant (and hence not invariant under $\phi$ ) and that $\omega_{0}\left(\zeta_{j}\right)=\omega_{0}\left(\zeta_{N-j}\right)$. This last assumption ensures that our dynamical system is time reversal invariant (TRI) with time reversal $\theta\left(\zeta_{j}\right)=\zeta_{N-j}$.

The entropy observable is $\mathcal{S}_{0}(\zeta)=-\log \omega_{0}(\zeta)$. The observable $\Sigma^{t}=\left(\mathcal{S}_{t}-\mathcal{S}_{0}\right) / t$ describes the mean entropy production rate over the time interval $[0, t]$. One easily verifies that for $t>0$,

$$
\omega_{0}\left(\Sigma^{t}\right)=-\frac{1}{t} S\left(\omega_{t}, \omega_{0}\right) \geq 0
$$

in accordance with the (finite time) second law of thermodynamics. The entropy production observable (or the phase space contraction rate) is defined by $\sigma(\zeta)=-\log \left(\omega_{1}(\zeta) / \omega_{0}(\zeta)\right)$ and satisfies $\Sigma^{t}=$ $t^{-1} \sum_{s=1}^{t} \sigma_{s}$.

The relation (2.1) holds without the TRI assumption. The TRI however allows to refine the second law as follows. Let $\mathcal{E}_{t \lambda}=\left\{\zeta \mid \Sigma^{t}(\zeta)=\lambda\right\}$ and $p^{t}(\lambda)=\omega_{0}\left(\mathcal{E}_{t \lambda}\right)$. An easy computation gives the celebrated Evans-Searles fluctuation relation

$$
p^{t}(-\lambda)=\mathrm{e}^{-\lambda t} p^{t}(\lambda) .
$$

This relation implies $\omega_{0}\left(\Sigma^{t}\right) \geq 0$ and is saying more: the negative values of the mean entropy production rate are exponentially suppressed in a universal manner.

The classical entropic functional is defined by

$$
e_{t}(\alpha)=\log \omega_{0}\left(\mathrm{e}^{-\alpha t \Sigma^{t}}\right)
$$

The symmetry

$$
e_{t}(\alpha)=e_{t}(1-\alpha)
$$

which holds for all $\alpha \in \mathbb{R}$, is an equivalent formulation of the fluctuation relation (2.2). Clearly, $e_{t}(0)=$ 0 , and hence $e_{t}(1)=0$ (this is sometimes called the Kawasaki identity [2]). The function $\alpha \mapsto e_{t}(\alpha)$ is convex, and $e_{t}^{\prime}(0)=-t \omega_{0}\left(\Sigma^{t}\right)$. The classical entropic functional satisfies

$$
e_{t}(\alpha)=\max _{\rho} S\left(\rho, \omega_{0}\right)-\alpha t \rho\left(\Sigma^{t}\right)
$$


and

$$
e_{t}(\alpha)=S_{\alpha}\left(\omega_{t}, \omega_{0}\right) .
$$

The functional $e_{t}(\alpha)$ can be also described in terms of Ruelle transfer operators. For $p \in[1, \infty$ [ we set $\|f\|_{p}^{p}=\sum_{\zeta}|f(\zeta)|^{p} \omega_{0}(\zeta)$ and define

$$
U_{p}(t) f=f_{-t} \mathrm{e}^{\frac{t}{p} \Sigma^{-t}}=f_{-t} \mathrm{e}^{-\frac{1}{p} \mathcal{S}_{-t}} \mathrm{e}^{\frac{1}{p} \mathcal{S}_{0}} .
$$

Then

$$
U_{p}\left(t_{1}+t_{2}\right)=U_{p}\left(t_{1}\right) U_{p}\left(t_{2}\right), \quad U_{p}(-t) f U_{p}(t) g=f_{t} g, \quad\left\|U_{p}(t) f\right\|_{p}=\|f\|_{p},
$$

i.e., $U_{p}$ is a group of isometries of the space $L^{p}\left(\mathcal{E}, \omega_{0}\right)$ which implements the dynamics. In terms of this group, one has

$$
e_{t}(\alpha)=\log \left\|U_{p / \alpha}(t) \mathbf{1}\right\|_{p}^{p}
$$

where $\mathbf{1}(\zeta)=1$.

The results described in this section extend under minimal regularity assumptions to an essentially arbitrary classical dynamical system [13].

\section{Quantum picture}

Consider a pair $(\mathcal{K}, H)$ where $\mathcal{K}$ is a finite dimensional Hilbert space and $H$ is a Hamiltonian. Observables are linear maps $A: \mathcal{K} \rightarrow \mathcal{K}$ (the identity map is denoted by $\mathbf{1}$ ) and states are strictly positive density matrices $\rho$ on $\mathcal{K}$. The observables evolve in time as $A_{t}=\mathrm{e}^{\mathrm{i} t H} A \mathrm{e}^{-\mathrm{i} t H}, t \in \mathbb{R}$, and states as $\rho_{t}=\mathrm{e}^{-\mathrm{i} t H} \rho \mathrm{e}^{\mathrm{i} t H}$. We write $\rho(A)=\operatorname{tr}(A \rho)$. The relative entropy of two states $S(\rho, \nu)=$ $\operatorname{tr}(\rho(\log \rho-\log \nu))$ satisfies $S(\rho, \nu) \leq 0$ and $S(\rho, \nu)=0$ iff $\rho=\nu$. The Rényi relative entropy is defined by $S_{\alpha}(\rho, \nu)=\log \operatorname{tr}\left(\rho^{\alpha} \nu^{1-\alpha}\right)$.

Our starting point is a quantum dynamical system $\left(\mathcal{K}, H, \omega_{0}\right)$, where $\omega_{0}$ is a given reference state. We assume that $\omega_{0}$ does not commute with $H$ and that the system is TRI, i.e., that there exists a complex conjugation on $\mathcal{K}$ that commutes with $H$ and $\omega_{0}$.

The entropy observable is $\mathcal{S}_{0}=-\log \omega_{0} . \quad \Sigma^{t}=\left(\mathcal{S}_{t}-\mathcal{S}_{0}\right) / t$ is the mean entropy production rate observable and for $t>0$ the finite time second law holds:

$$
\omega_{0}\left(\Sigma^{t}\right)=-\frac{1}{t} S\left(\omega_{t}, \omega_{0}\right) \geq 0 .
$$

The entropy production observable (or the quantum phase space contraction rate) is $\sigma=-\mathrm{i}\left[H, \log \omega_{0}\right]$ and $\Sigma^{t}=t^{-1} \int_{0}^{t} \sigma_{s} \mathrm{~d} s$.

To aid the reader we describe one concrete physical setup. Consider two quantum dynamical systems $\left(\mathcal{K}_{l / r}, H_{l / r}, \omega_{l / r}\right)$, colloquially called the left and the right. Assume that initially the $l / r$ system is in thermal equilibrium at inverse temperature $\beta_{l / r}$, i.e., that $\omega_{l / r}=\mathrm{e}^{-\beta_{l / r} H_{l / r}} / Z_{\ell / r}$. Let $\mathcal{K}=\mathcal{K}_{\ell} \otimes \mathcal{K}_{r}$, $\omega_{0}=\omega_{l} \otimes \omega_{r}$, and $H=H_{l}+H_{r}+V$, where $V$ describes the interaction between the left and the right system. In this case

$$
\sigma=-\beta_{l} \Phi_{l}-\beta_{r} \Phi_{r},
$$


where $\Phi_{l / r}=\mathrm{i}\left[H_{l / r}, V\right]$ satisfies

$$
H_{l / r t}-H_{l / r}=-\int_{0}^{t} \Phi_{l / r s} \mathrm{~d} s
$$

and describes the energy flux out of the $l / r$ system.

We now turn to quantum fluctuation relations. TRI implies that the spectrum of $\Sigma^{t}$ is symmetric w.r.t. the origin. If $P_{t \lambda}$ is the spectral projection of $\Sigma^{t}$ onto $\lambda$ and $p^{t}(\lambda)=\omega_{0}\left(P_{\lambda t}\right)$, then the direct quantization of the fluctuation relation (2.2) is

$$
p^{t}(-\lambda)=\mathrm{e}^{-\lambda t} p^{t}(\lambda)
$$

Setting

$$
e_{t}(\alpha)=\log \omega_{0}\left(\mathrm{e}^{-\alpha t \Sigma^{t}}\right)
$$

one easily verifies that (3.10) holds iff $e_{t}(\alpha)=e_{t}(1-\alpha)$. However, one can show that the quantum Kawasaki identity $e_{t}(1)=0$ holds for all $t$ iff $\left[H, \omega_{0}\right]=0$ and so the direct quantization of (2.2) fails. The standard route of observable quantization does not lead to quantum fluctuation relations.

The following family of quantum entropic functionals indexed by $p \in[1, \infty]$ was introduced in [12]:

$$
e_{p t}(\alpha)= \begin{cases}\log \operatorname{tr}\left(\left[\mathrm{e}^{-\frac{1-\alpha}{p} \mathcal{S}_{0}} \mathrm{e}^{-\frac{2 \alpha}{p} \mathcal{S}_{t}} \mathrm{e}^{-\frac{1-\alpha}{p} \mathcal{S}_{0}}\right]^{\frac{p}{2}}\right) & \text { if } 1 \leq p<\infty, \\ \log \operatorname{tr}\left(\mathrm{e}^{-(1-\alpha) \mathcal{S}_{0}-\alpha \mathcal{S}_{t}}\right) & \text { if } p=\infty\end{cases}
$$

To motivate these functionals, note that

$$
e_{\infty t}(\alpha)=\max _{\rho} S\left(\rho, \omega_{0}\right)-\alpha t \rho\left(\Sigma^{t}\right)
$$

and so $e_{\infty t}(\alpha)$ is the quantization of the variational formula (2.5). We also have

$$
e_{2 t}(\alpha)=S_{\alpha}\left(\omega_{t}, \omega\right)
$$

and so $e_{2 t}(\alpha)$ is the quantization of (2.6). Regarding the other functionals, we need to introduce first the quantization of Ruelle's transfer operators. The quantization of the usual $L^{p}$-norm is the Araki-Masuda $L^{p}$-norm

$$
\|A\|_{p}^{p}=\operatorname{tr}\left(\left|A \omega_{0}^{\frac{1}{p}}\right|^{p}\right) .
$$

The classical transfer operators (2.7) are quantized as

$$
U_{p}(t) A=A_{-t} \mathrm{e}^{-\frac{1}{p} \mathcal{S}_{-t}} \mathrm{e}^{\frac{1}{p} \mathcal{S}_{0}} .
$$

They satisfy

$$
U_{p}\left(t_{1}+t_{2}\right)=U_{p}\left(t_{1}\right) U_{p}\left(t_{2}\right), \quad U_{p}(-t) A U_{p}(t) B=A_{t} B, \quad\left\|U_{p}(t) A\right\|_{p}=\|A\|_{p},
$$

and for $p \in[1, \infty[$,

$$
e_{p t}(\alpha)=\log \left\|U_{p / \alpha}(t) \mathbf{1}\right\|_{p}^{p}
$$


Hence, the functionals $e_{p t}(\alpha)$ for $p \in[1, \infty[$ arise as the quantization of (2.8).

The functionals $e_{p t}(\alpha)$ have the following properties. The symmetry

$$
e_{p t}(\alpha)=e_{p t}(1-\alpha)
$$

holds for all $p$ and $\alpha$ and implies the quantum Kawasaki identity $e_{p t}(1)=e_{p t}(0)=0$. The function $\alpha \rightarrow e_{p t}(\alpha)$ is convex and the function $p \mapsto e_{p t}(\alpha)$ is continuous and decreasing. For all $p, e_{p t}^{\prime}(0)=$ $-t \omega_{0}\left(\Sigma^{t}\right)$.

The functional $e_{2 t}(\alpha)$ has appeared previously in the literature in two seemingly unrelated contexts. In [14] this functional was related to the fundamental concept of Full Counting Statistics (FCS) associated to the repeated measurement protocol of the entropy flow. Let $\mathcal{S}_{0}=\sum_{\lambda} \lambda P_{\lambda}$ be the spectral resolution of the entropy observable. The probability that a measurement of $\mathcal{S}_{0}$ at time $t=0$ (when the system is in the state $\left.\omega_{0}\right)$ yields $\lambda$ is $\omega_{0}\left(P_{\lambda}\right)$. After the measurement, the system is in the reduced state $\omega_{0} P_{\lambda} / \omega_{0}\left(P_{\lambda}\right)$ which evolves in time as $\mathrm{e}^{-\mathrm{i} t H} \omega_{0} P_{\lambda} \mathrm{e}^{\mathrm{i} t H} / \omega_{0}\left(P_{\lambda}\right)$. Denoting $\lambda^{\prime}$ the outcome of a second measurement of $\mathcal{S}_{0}$ at a later time $t>0$, the joint probability distribution of these two measurements is $\operatorname{tr}\left(\mathrm{e}^{-\mathrm{i} t H} \omega_{0} P_{\lambda} \mathrm{e}^{\mathrm{i} t H} P_{\lambda^{\prime}}\right)$. It follows that the probability of observing a mean rate of entropy change $\phi=\left(\lambda^{\prime}-\lambda\right) / t$ is

$$
\mathbb{P}_{t}(\phi)=\sum_{\lambda^{\prime}-\lambda=t \phi} \operatorname{tr}\left(\mathrm{e}^{-\mathrm{i} t H} \omega_{0} P_{\lambda} \mathrm{e}^{\mathrm{i} t H} P_{\lambda^{\prime}}\right)
$$

The discrete probability measure $\mathbb{P}_{t}$ is the FCS for the operationally defined entropy change over the time interval $[0, t]$ as specified by the above measurement protocol. It is easy to verify that the $e_{2 t}(\alpha)$ is the cumulant generating function for the FCS, i.e.,

$$
e_{2 t}(\alpha)=\log \sum_{\phi} \mathrm{e}^{-t \alpha \phi} \mathbb{P}_{t}(\phi)
$$

and the symmetry $e_{2 t}(\alpha)=e_{2 t}(1-\alpha)$ yields the fluctuation relation

$$
\mathbb{P}_{t}(-\phi)=\mathrm{e}^{-t \phi} \mathbb{P}_{t}(\phi)
$$

In [20] the functional $e_{2 t}(\alpha)$ was motivated by the algebraic characterization of the Zubarev dynamical ensemble [21]. Let $\mathcal{O}$ be the vector space of all linear maps $A: \mathcal{K} \rightarrow \mathcal{K}$ equipped with the inner product $\langle A, B\rangle=\operatorname{tr}\left(A^{*} B\right)$. The relative modular operator

$$
\Delta_{\omega_{t} \mid \omega_{0}}(A)=\omega_{t} A \omega_{0}^{-1}
$$

is a strictly positive operator on the Hilbert space $(\mathcal{O},\langle\cdot, \cdot\rangle)$. Let $Q_{t}$ be the spectral measure of $-\frac{1}{t} \log \Delta_{\omega_{t} \mid \omega_{0}}$ for the vector $\omega_{0}^{1 / 2} \in \mathcal{O}$. Then

$$
e_{2 t}(\alpha)=\log \sum_{s} \mathrm{e}^{-\alpha t s} Q_{t}(s),
$$

and in particular, $\mathbb{P}_{t}=Q_{t}$. This identification provides a striking link between the FCS and modular theory with far reaching implications. 


\section{Research program}

In telegraphic terms, our research program can be outlined as follows.

(a) The first part of the program, carried out in [10], deals with development of the finite time theory of entropic functionals in a general dynamical system setting. In the classical case this step is relatively easy [13]. The quantum case is substantially more difficult and the full machinery of Tomita-Takesaki modular theory is required. The functionals $e_{p t}(\alpha)$ and quantum Ruelle transfer operators are based on the Araki-Masuda theory of non-commutative $L^{p}$-spaces [1]. The step (a) can be viewed as an abstract unraveling of the mathematical structures underlying the entropic functionals. These structures turn out to be of considerable conceptual and computational importance.

(b) This step concerns the existence and regularity properties of the limiting functionals

$$
e_{p+}(\alpha)=\lim _{t \rightarrow \infty} \frac{1}{t} e_{p t}(\alpha)
$$

and is a difficult ergodic type problem that can be studied only in the context of concrete models. The existing results cover open spin-fermion and spin-boson systems (the proofs are based on the analysis of resonances of quantum Ruelle transfer operators, see also [16] for a pioneering work on the subject), and open locally interacting fermionic systems (the proofs are based on $C^{*}$-scattering techniques). In some special cases (like the $X Y$ chain) the functionals $e_{p+}(\alpha)$ can be expressed in closed form and analyzed in great detail. The step (b) of the program has been carried out in a series of papers and is a joint work with B. Landon, Y. Ogata, A. Panati, Y. Pautrat, and M. Westrich. It remains a challenge to extend these results to a wider class of models. We emphasize that the step (b) is meaningful only in the context of infinitely extended models (in other words, the thermodynamic limit must precede the large time limit). The thermodynamic limit is also needed for the physical interpretation of the finite time quantum entropic functionals of infinitely extended systems.

(c) The Legendre transform of the limiting functional $e_{2+}(\alpha)$ is the rate function describing the large deviation fluctuations of the full counting statistics as $t \rightarrow \infty$. In the linear regime and under suitable regularity assumptions the existence of $e_{2+}(\alpha)$ also implies the central limit theorem for the full counting statistics. For open quantum systems and in the linear regime (near equilibrium and for small $\alpha$ ) all

functionals $e_{p+}(\alpha)$ reduce to Green-Kubo formulas for heat/charge currents. However, the development of linear response theory that goes beyond open quantum systems and allows for general thermodynamical/mechanical forces can be based only on the functional $e_{\infty+}(\alpha)$ (see [5, 13] for the results in the classical case that motivated this development). The functionals $e_{p+}(\alpha)$, together with symmetries $e_{p+}(\alpha)=e_{p+}(1-\alpha)$, can be viewed as extensions of the fluctuation-dissipation theorem to the far from equilibrium regime.

(d) The functional $e_{2+}(\alpha)$ coincides with the Chernoff error exponent for the quantum hypothesis testing of the arrow of time and this links quantum hypothesis testing, a rapidly developing branch of quantum information theory, to non-equilibrium statistical mechanics. This connection has been explored in [11]. (e) We have only discussed the entropic functionals defined with respect to the reference (initial) state of the system (they are sometimes called Evans-Searles type functionals). The Gallavotti-Cohen type entropic functionals are defined with respect to the non-equilibrium steady state (NESS) (the state that the infinitely extended system reaches in the large time limit). The Gallavotti-Cohen type functionals are considerably more technical to introduce and study, and in the quantum case have a somewhat delicate physical interpretation. The Principle of Regular Entropic Fluctuations (PREF) introduced in 
$[13,10]$ asserts that under normal conditions the limiting entropic functional of the Evans-Searles and Gallavoti-Cohen type are identical. Since under normal conditions the NESS is singular with respect to the reference state, the PREF can be viewed as a strong ergodic property of the physical model under consideration.

(f) The developement of nonequilibrium statistical mechanics of open quantum systems in the Markovian approximation started with the pioneering work of Lebowitz and Spohn [15]. Since the Markovian approximation is often the only technically accessible way to describe an open system, it is also important to develop a general theory of entropic fluctuations in this framework. The first attempts in this direction are due to Dereziński, De Roeck and Maes [17, 3]. In [9], we derive fluctuation relations starting from structural properties of the generator (Lindbladian) of the Markovian dynamics.

\section{Remarks}

Non-equilibrium statistical mechanics is a difficult subject and for many years our theoretical understanding has been restricted to linear regime near equilibrium (linear response theory, the fluctuationdissipation theorem). There are good reasons for this: the richness and variety of non-equilibrium phenomena indicate that far from equilibrium physics may tolerate very few universal constraints. The recently discovered fluctuation relations of Evans-Searles and Gallavotti-Cohen are two universally valid constraints that hold far from equilibrium and reduce to linear response near equilibrium. These insights and subsequent developments (see [13] for references) have dramatically altered our understanding of classical non-equilibrium statistical mechanics. The extensions of fluctuation relations to quantum do-

main have led to further surprises that still remain to be fully explored. The research program outlined in this note is a first step in this direction.

\section{References}

[1] Araki H., Masuda T.: Positive cones and $L^{p}$-spaces for von Neumann algebras. Publ. RIMS, Kyoto Univ. 18, 339-411 (1982).

[2] Carberry D.M., Williams S.R., Wang G.M., Sevick E.M., Evans D.J.: The Kawasaki identity and the fluctuation theorem. J. Chem. Phys. 121, 8179-8182 (2004).

[3] Dereziński J., De Roeck W., Maes C.: Fluctuations of quantum currents and unravelings of master equations. J. Stat. Phys. 131, 341-356 (2008).

[4] Evans D.J., Searles D.J.: Equilibrium microstates which generate second law violating steady states. Phys Rev. E 50, 1645-1648 (1994).

[5] Gallavotti G.: Chaotic hypothesis: Onsager reciprocity and fluctuation-dissipation theorem. J. Stat. Phys. 84, 899-925 (1996).

[6] Gallavotti G., Cohen E.G.D.: Dynamical ensembles in nonequilibrium statistical mechanics. Phys. Rev. Lett. 74, 2694-2697 (1995). 
[7] Jakšić V., Pillet C.-A.: On entropy production in quantum statistical mechanics. Commun. Math. Phys. 217, 285-293 (2001).

[8] Jakšić V., Pillet C.-A.: Mathematical theory of non-equilibrium quantum statistical mechanics. J. Stat. Phys. 108, 787-829 (2002).

[9] Jakšić V., Pillet C.-A., Westrich M.: Entropic fluctuations for quantum dynamical semigroups. In preparation.

[10] Jakšić V., Ogata Y., Pillet C.-A.: Entropic fluctuations in statistical mechanics II. Quantum dynamical systems. In preparation.

[11] Jakšić V., Ogata Y., Pillet C.-A., Seiringer R.: Hypothesis testing and non-equilibrium statistical mechanics. Rev. Math. Phys 24, 1-67 (2012).

[12] Jakšić V., Ogata Y., Pautrat Y., Pillet C.-A.: Entropic fluctuations in quantum statistical mechanics. An Introduction. In Quantum Theory from Small to Large Scales. Lecture Notes of the Les Houches Summer School Volume 95, August 2010. Oxford University Press, USA (2012).

[13] Jakšić V., Pillet C.-A., Rey-Bellet L.: Entropic fluctuations in statistical mechanics I. Classical dynamical systems. Nonlinearity 24, 699-763 (2011).

[14] Kurchan J.: A quantum Fluctuation theorem. arXiv:cond-mat/0007360v2 (2000).

[15] Lebowitz J.L., Spohn H.: Irreversible thermodynamics for quantum systems weakly coupled to thermal reservoirs. Adv. Chem. Phys. 38, 109-142 (1978).

[16] De Roeck W.: Large deviation generating function for currents in the Pauli-Fierz model. Rev. Math. Phys. 21, 549-585 (2009).

[17] De Roeck W., Maes C.: Steady state fluctuations of the dissipated heat for a quantum stochastic model. Rev. Math. Phys. 18, 619-653 (2006).

[18] Ruelle D.: Natural nonequilibrium states in quantum statistical mechanics. J. Stat. Phys. 98, 57-75 (2000).

[19] Ruelle D.: Entropy production in quantum spin systems. Commun. Math. Phys. 224, 3-16 (2001).

[20] Tasaki S., Matsui T.: Fluctuation theorem, non-equilibrium steady states and MacLennan-Zubarev ensembles of a class of large quantum systems. Quantum Prob. White Noise Anal. 17, 100-119 (2003).

[21] Zubarev D.N.: Nonequilibrium Statistical Thermodynamics. Consultants, New York, (1974). 